\title{
Microvascular obstruction in acute myocardial infarction: an old and unsolved mystery
}

\author{
Mădălin Constantin Marc ${ }^{1,2}$, Adrian Corneliu Iancu ${ }^{1,2}$, Şerban Bălănescu ${ }^{3}$, \\ Mihaela Ioana Dregoesc ${ }^{1,2}$
}

1) Department of Cardiology, Iuliu Hatieganu University of Medicine and Pharmacy, Cluj-Napoca, Romania

2) "Niculae Stãncioiu” Heart Institute, Cluj-Napoca, Romania

3) "Carol Davila" University of Medicine and Pharmacy, Elias University Hospital, Bucharest, Romania

\begin{abstract}
In the setting of acute myocardial infarction, flow restoration in the culprit epicardial coronary artery is not synonymous with efficient reperfusion. Microvascular obstruction occurs in $50 \%$ of cases and represents a predictor of a long-term unfavorable outcome. Its prevalence has remained constant in recent years despite various treatment attempts. However, the success of targeted therapies could be mainly a problem of timing.

Recent data bring evidence with regard to the role of pre-procedural distal embolization and highlight the relation between distal embolism, microvascular obstruction and intramyocardial inflammation. As a result, early detection of microvascular injury represents the first step in the development of targeted, individualized therapeutic approaches.

In this context, the identification of new invasive surrogate parameters for the timely assessment and quantification of microvascular obstruction in the catheterization laboratory has become an important subject of current research. Among these, coronary wedge pressure is the most practical and revealing in the setting of primary percutaneous coronary intervention. It may offer comprehensive details on the mechanisms of microvascular injury and may therefore offer guidance for appropriate treatment selection.
\end{abstract}

Keywords: acute myocardial infarction, percutaneous coronary intervention, microvascular obstruction, intramyocardial hemorrhage, coronary wedge pressure

\section{Introduction}

Reopening an occluded or stenotic epicardial coronary artery by primary percutaneous coronary intervention (PCI) is accepted as the preferred treatment for patients with acute myocardial infarction (AMI). However, despite successful opening of the culprit epicardial vessel, microvascular obstruction (MVO) occurs in about $50 \%$ of cases. At a microcirculatory level, the failure of reperfusion is linked with an unfavorable evolution at long term follow-up.

In a study by Ndrepepa [1], MVO was an independent predictor of mortality and correlated with the extent of necrosis. Actually, the presence of MVO is a powerful predictor of functional recovery and in the end, of left ventricular remodeling and heart failure [2-5]. None of the recent trials that aimed to protect the microcirculation during PCI demonstrated any benefit.

\section{Mechanisms of microvascular obstruction}

The mechanisms of myocardial injury are initiated prior to the occlusion of the epicardial coronary artery. They are represented by the susceptibility of the micro-vessels for endothelial dysfunction (e.g. diabetes mellitus), and by the distal propagation of intravascular inflammation to the myocardium. Kramer hypothesized [6] that embolism occurs before the clinical onset of ST-segment elevation myocardial infarction (STEMI) and before vessel occlusion. Inflammation starts in vulnerable atherosclerotic plaques and involves the friable superimposed thrombus. Following distal thrombus embolization, inflammatory cells reach the microcirculation. This process can be defined as pre-procedural MVO, and is linked to higher mortality at long term follow-up. It occurs in about $50 \%$ of patients with STEMI and it is characterized 
by the presence of old thrombus. Old thrombus is considered to have developed for more than one day and is infiltrated with inflammatory cells [7]. Neutrophil aggregates and coronary neutrophil extracellular trap burden from aspirated thrombotic material are associated with impaired coronary microcirculation [8]. In this stage, cellular plugging and embolization have been recorded. These findings are similar to those observed following percutaneous revascularization. The final stage, most severe form of MVO is represented by intramyocardial edema and hemorrhage.

The extent and importance of distal embolization during PCI is a matter of continuous debate. Until now, all of the prophylactic techniques and tools used in clinical trials failed to prove any benefit. Both EMERALD and DEDICATION trials, which used anti-embolic devices during PCI, had disappointing results $[9,10]$. Furthermore, none of the large thrombus aspiration studies and large recent meta-analyses [11-14] have proven to be of any benefit on MVO. All these disappointing therapeutic attempts raised many question with regard to the importance of PCI induced embolism. These negative results led to the conclusion that distal thromboembolism during PCI might not be sufficient to produce extensive injury and might be only a small contributor to the resulting MVO.

Several therapeutic strategies are currently designed to target capillary integrity during PCI, as interstitial edema and hemorrhage have recently been demonstrated as predictors of an unfavorable evolution. Hypoxia and inflammatory induced disruption of the endothelial barrier leads to the loss of vessel integrity and results in the extravasation of erythrocytes and to consequent intramyocardial hemorrhage (IMH). An important issue arises from the sudden increase in distal pressure after stenting, which theoretically contributes to the rupture of capillary walls.

\section{Detection of microvascular obstruction and therapeutic targets}

Except for ST-segment resolution (STR) and myocardial blush grade (MBG), all the other MVO detection techniques are usually performed 3 to 5 days post-PCI. This time interval is well outside of a possible therapeutic window, especially if the onset of MVO takes place days before presentation. Both STR and MBG have low sensitivity and specificity for the accurate detection of MVO and offer discordant results in almost $40 \%$ of patients $[15,16]$.

The most important issue with regard to an early therapeutic approach consists in the early detection of microvascular injury. Invasive surrogate parameters can be determined during primary PCI and may represent a solution to this problem. Coronary flow reserve (CFR), diastolic deceleration time (DDT) and the presence of systolic flow reversal (SFR) can be measured using Doppler and thermodilution techniques. A CFR value of less than 2.0 has a sensitivity of $79 \%$ for the detection of MVO and $80 \%$ for the detection of IMH. However, the specificity for both MVO and IMH is of only $34 \%$ [17]. The index of microvascular resistance (IMR) is one of the most important surrogate parameters that define MVO. This invasive marker has recently been shown to be an independent predictor of IMH, a consequence of severe microvascular damage. However the determination of IMR is demanding and time-consuming during primary PCI. Some studies have demonstrated a good correlation between IMR at the time of PCI and cardiac magnetic resonance (CMR) imaging data. Patients with IMR higher than $40 \mathrm{U}$ were more likely to have MVO and IMH [18-21]. However, some studies had opposite results with regard to the relationship between IMR and CMR detected MVO [16,22,23]. What is more, one recent study has shown that Zero-Flow Pressure (Pzf) is a more sensitive measure of microvascular function as compared with IMR [24]. Both coronary wedge pressure (CWP) and Pzf are mainly dependent on the extent of external microcirculatory compression by edema and IMH. CWP measurement is a more practical alternative to Pzf and it is not time consuming $[25,26]$.

But why is the early detection of IMH so important? CMR is the gold standard in detecting and quantifying IMH, but it can be performed only 3-5 days after primary PCI. On the other hand, CWP is measured prior to revascularization [5] and can provide guidance for a new therapeutic approach. One therapeutic option is the early administration of antiinflammatory or chelation therapy. Moreover, since capillary overpressurization is important for the occurrence of myocardial edema and IMH, primary PCI technique can be modified as to prevent an uncontrolled pressure increase. Several questions arise. Is predilatation in this setting a tool to prevent the abrupt increase in distal pressure? Is postconditioning a more important mechanism than previously suspected? Several important clinical trials [27-30] have shown an increase in myocardial salvage ratio as evaluated by CMR and a decrease in enzyme leakage in patients undergoing ischemic post-conditioning. The technique of postconditioning modulates reperfusion pressure and its effect on capillarity integrity. IMH leads to residual myocardial iron, which represents a source of prolonged inflammation and has an impact on adverse left ventricular remodeling. In an experimental model, Kali $[30,31]$ showed that IMH provided a source of prolonged inflammatory burden in the chronic phase.

\section{Current treatment}

The goal of any new therapy for STEMI is the reduction in left ventricular remodeling and mortality. In current times, the treatment of acute myocardial infarction is effective through PCI reperfusion. A further reduction in mortality should be accomplished by targeting MVO. However, any new treatment necessitates large sample sized trials, with long-term follow-up. Until now, these are unfortunately missing. Briefly, early diagnostic imposes early treatment and this can happen only in the catheterization 
laboratory. Intracoronary administration of drugs through the guiding catheter has not proved any benefit on MVO and this is the easiest and most used approach. In this technique the administered drugs fail to rich the microvessels because of the aortic reflow, of collateral stealing and finally of the inability to cross the occluded microvessels. The downstream intracoronary administration avoids these disadvantages, creates high distal drug concentrations and increases contact time with the myocardial interstitium. Administration through microcatheters after wiring the culprit lesions and before stenting is an attractive alternative. There are only a few substances that can fulfill the necessary characteristics for the effective treatment of MVO. These include the antithrombotic and the lythic effect on thrombus, as well as the antiinflammatory capacities. Glycoprotein IIbIIIa inhibitors (GPIIb/IIIa) like abciximab, eptifibatide and tirofiban gather all these qualities [32]. GPIIb/IIIa inhibitors abrogate the NF-kB activation and implicate that these antithrombotic drugs also exercise antiinflammatory effects. GP IIb/IIIa antagonists inhibit the sCD40 ligand release from activated platelets into the circulation. Plateletderived $\mathrm{sCD} 40$ ligand plays a detrimental role through the induction and release of proinflammatory cytokines $[33,34]$. There are only small studies testing GPIIbIIIa downstream intracoronary approach, but none of them selected high risk MVO patients [35,36].

The algorithm we propose consists in pre-PCI measurement of CWP, followed by downstream GPIIbIIIa inhibitors administration in patients with high CWP.

\section{Future directions}

The pathogenetic mechanisms of MVO represent a promising target in the individualized treatment of patients with STEMI. In addition, ongoing techniques for the assessment and quantification of MVO in the cardiac catheterization laboratory will provide early and optimal tools to evaluate and quantify the extent of microvascular injury. What is more, these invasive parameters will offer guidance for the use of potential new therapies. A CWP cut-off value for the prediction of MVO could offer the premises for an early therapeutic approach. While an elevated CWP could define interstitial edema and hemorrhage and could establish an indication for antiinflammatory and chelation therapy, a tall systolic "V" wave on the same pressure curve could impose downstream intracoronary administration of glycoprotein IIbIIIa inhibitors. Among these, abciximab is the most promising through its antiinflammatory and lythic effects on thrombotic emboli. The final objective would be to test these new therapies in relation to the prevention of left ventricular remodeling, heart failure and consequent mortality, the actual therapeutic goals and the major efficacy endpoints.

\section{References}

1. Ndrepepa G, Tiroch K, Fusaro M, Keta D, Seyfarth M, Byrne RA, et al. 5-year prognostic value of no-reflow phenomenon after percutaneous coronary intervention in patients with acute myocardial infarction. J Am Coll Cardiol. 2010;55:2383-2389.

2. Lombardo A, Niccoli G, Natale L, Bernardini A, Cosentino $\mathrm{N}$, Bonomo $\mathrm{L}$, et al. Impact of microvascular obstruction and infarct size on left ventricular remodeling in reperfused myocardial infarction: a contrast-enhanced cardiac magnetic resonance imaging study. Int $\mathrm{J}$ Cardiovasc Imaging. 2012;28:835-842.

3. Weir RA, Murphy CA, Petrie CJ, Martin TN, Balmain S, Clements $\mathrm{S}$, et al. Microvascular obstruction remains a portent of adverse remodeling in optimally treated patients with left ventricular systolic dysfunction after acute myocardial infarction. Circ Cardiovasc Imaging. 2010;3:360-367.

4. de Waha S, Desch S, Eitel I, Fuernau G, Lurz P, Leuschner A, et al. Relationship and prognostic value of microvascular obstruction and infarct size in ST-elevation myocardial infarction as visualized by magnetic resonance imaging. Clin Res Cardiol. 2012;101:487-495.

5. Marc MC, Iancu AC, Ober CD, Homorodean C, Bãlãnescu Ş, Sitar AV, et al. Pre-revascularization coronary wedge pressure as marker of adverse long-term left ventricular remodelling in patients with acute ST-segment elevation myocardial infarction. Sci Rep. 2018; 8:1897.

6. Kramer MC, van der Wal AC, Koch KT, Ploegmakers JP, van der Schaaf RJ, Henriques JP, et al. Presence of older thrombus is an independent predictor of long-term mortality in patients with ST-elevation myocardial infarction treated with thrombus aspiration during primary percutaneous coronary intervention. Circulation. 2008;118:1810-1816.

7. Yunoki K, Naruko T, Inoue T, Sugioka K, Inaba M, Iwasa $\mathrm{Y}$, et al. Relationship of thrombus characteristics to the incidence of angiographically visible distal embolization in patients with ST-segment elevation myocardial infarction treated with thrombus aspiration. JACC Cardiovasc Interv. 2013;6:377-385.

8. Mangold A, Alias S, Scherz T, Hofbauer T, Jakowitsch J, Panzenböck A, et al. Coronary neutrophil extracellular trap burden and deoxyribonuclease activity in ST-elevation acute coronary syndrome are predictors of ST-segment resolution and infarct size. Circ Res. 2015;116:1182-1192.

9. Stone GW, Webb J, Cox DA, Brodie BR, Qureshi M, Kalynych A, et al. Distal microcirculatory protection during percutaneous coronary intervention in acute ST-segment elevation myocardial infarction: a randomized controlled trial. JAMA. 2005;293:1063-1072.

10. Kelbaek H, Terkelsen CJ, Helqvist S, Lassen JF, Clemmensen P, Kløvgaard L, et al. Randomized comparison of distal protection versus conventional treatment in primary percutaneous coronary intervention: the drug elution and distal protection in ST-elevation myocardial infarction (DEDICATION) trial. J Am Coll Cardiol. 2008;51:899-905.

11. Jolly SS, Cairns JA, Yusuf S, Rokoss MJ, Gao P, Meeks B, et al. Outcomes after thrombus aspiration for ST elevation myocardial infarction: 1-year follow-up of the prospective randomised TOTAL trial. Lancet. 2016;387:127-135.

12. Fröbert O, Lagerqvist B, Olivecrona GK, Omerovic E, Gudnason T, Maeng M, et al. Thrombus aspiration during 
ST-segment elevation myocardial infarction. N Engl J Med. 2013;369:1587-1597.

13. Jolly SS, James S, Džavik V, Cairns JA, Mahmoud KD, Zijlstra F, et al. Thrombus Aspiration in ST-SegmentElevation Myocardial Infarction: An Individual Patient Meta-Analysis: Thrombectomy Trialists Collaboration. Circulation. 2017;135:143-152.

14. Mahmoud KD, Zijlstra F. Thrombus aspiration in acute myocardial infarction. Nat Rev Cardiol. 2016;13:418-428.

15. Sorajja P, Gersh BJ, Costantini C, McLaughlin MG, Zimetbaum P, Cox DA, et al. Combined prognostic utility of ST-segment recovery and myocardial blush after primary percutaneous coronary intervention in acute myocardial infarction. Eur Heart J. 2005;26:667-674.

16. Nijveldt R, Beek AM, Hirsch A, Stoel MG, Hofman MB, Umans VA, et al. Functional recovery after acute myocardial infarction: comparison between angiography, electrocardiography, and cardiovascular magnetic resonance measures of microvascular injury. J Am Coll Cardiol. 2008;52:181-189.

17. Carrick D, Haig C, Carberry J, May VTY, McCartney $\mathrm{P}$, Welsh $\mathrm{P}$, et al. Microvascular resistance of the culprit coronary artery in acute ST-elevation myocardial infarction. JCI Insight. 2016;1:e85768.

18. McGeoch R, Watkins S, Berry C, Steedman T, Davie A, Byrne $\mathrm{J}$, et al. The index of microcirculatory resistance measured acutely predicts the extent and severity of myocardial infarction in patients with ST-segment elevation myocardial infarction. JACC Cardiovasc Interv. 2010;3:715-722.

19. Yoo SH, Yoo TK, Lim HS, Kim MY, Koh JH. Index of microcirculatory resistance as predictor for microvascular functional recovery in patients with anterior myocardial infarction. J Korean Med Sci. 2012;27:1044-1050.

20. Ahn SG, Hung OY, Lee JW, Lee JH, Youn YJ, Ahn MS, et al. Combination of the thermodilution-derived index of microcirculatory resistance and coronary flow reserve is highly predictive of microvascular obstruction on cardiac magnetic resonance imaging after ST-segment elevation myocardial infarction. JACC Cardiovasc Interv. 2016;9:793-801.

21. Cuculi F, De Maria GL, Meier P, Dall'Armellina E, de Caterina AR, Channon KM, et al. Impact of microvascular obstruction on the assessment of coronary flow reserve, index of microcirculatory resistance, and fractional flow reserve after ST-segment elevation myocardial infarction. J Am Coll Cardiol. 2014;64:1894-1904.

22. Hoole SP, Jaworski C, Brown AJ, McCormick LM, Agrawal B, Clarke SC, et al. Serial assessment of the index of microcirculatory resistance during primary percutaneous coronary intervention comparing manual aspiration catheter thrombectomy with balloon angioplasty (IMPACT study): a randomised controlled pilot study. Open Heart. 2015;2:e000238.

23. Bulluck H, Foin N, Cabrera-Fuentes HA, Yeo KK, Wong AS, Fam JM, et al. Index of microvascular resistance and microvascular obstruction in patients with acute myocardial infarction. JACC Cardiovasc Interv. 2016;9:2172-2174.

24. Patel N, Petraco R, Dall'Armellina E, Kassimis G, De Maria GL, Dawkins S, et al. Zero-Flow Pressure Measured
Immediately After Primary Percutaneous Coronary Intervention for ST-Segment Elevation Myocardial Infarction Provides the Best Invasive Index for Predicting the Extent of Myocardial Infarction at 6 Months: An OxAMI Study (Oxford Acute Myocardial Infarction). JACC Cardiovasc Interv. 2015;8:1410-1421.

25. Echavarria-Pinto M, Serruys PW, Garcia-Garcia HM, Broyd C, Cerrato E, MacAya C, et al. Use of intracoronary physiology indices in acute coronary syndromes. Interventional Cardiology. 2015;7:483-495.

26. Iancu AC, Rafiroiu D, Marc M. Is Coronary Wedge Pressure a Technique to Identify High-Risk Patients Who May Benefit From Alternative Treatment in Acute Myocardial Infarction?: Is This The Next Step? JACC Cardiovasc Interv. 2016;9:104-105.

27. Lønborg J, Kelbaek H, Vejlstrup N, Jørgensen E, Helqvist $S$, Saunamäki K, et al. Cardioprotective effects of ischemic postconditioning in patients treated with primary percutaneous coronary intervention, evaluated by magnetic resonance. Circ Cardiovasc Interv. 2010;3:34-41.

28. Staat P, Rioufol G, Piot C, Cottin Y, Cung TT, L'Huillier I, et al. Postconditioning the human heart. Circulation. 2005;112:2143-2148.

29. Thibault H, Piot C, Ovize M. Postconditioning in man. Heart Fail Rev. 2007;12:245-248.

30. Kali A, Kumar A, Cokic I, Tang RL, Tsaftaris SA, Friedrich $\mathrm{MG}$, et al. Chronic manifestation of postreperfusion intramyocardial hemorrhage as regional iron deposition: a cardiovascular magnetic resonance study with ex vivo validation. Circ Cardiovasc Imaging. 2013;6:218-228.

31. Kali A, Cokic I, Tang R, Dohnalkova A, Kovarik L, Yang HJ, et al. Persistent microvascular obstruction after myocardial infarction culminates in the confluence of ferric iron oxide crystals, proinflammatory burden, and adverse remodeling. Circ Cardiovasc Imaging. 2016;9. pii: e004996.

32. Speich HE, Earhart AD, Hill SN, Cholera S, Kueter TJ, Smith $\mathrm{JN}$, et al. Variability of platelet aggregate dispersal with glycoprotein IIb-IIIa antagonists eptifibatide and abciximab. J Thromb Haemost. 2009;7:983-991.

33. Welt FG, Rogers SD, Zhang X, Ehlers R, Chen Z, NannizziAlaimo L, et al. GP IIb/IIIa inhibition with eptifibatide lowers levels of soluble CD40L and RANTES after percutaneous coronary intervention. Catheter Cardiovasc Interv. 2004;61:185-189.

34. Furman MI, Krueger LA, Linden MD, Fox ML, Ball SP, Barnard MR, et al. GPIIb-IIIa antagonists reduce thromboinflammatory processes in patients with acute coronary syndromes undergoing percutaneous coronary intervention. J Thromb Haemost. 2005;3:312-320.

35. Bellandi F, Maioli M, Gallopin M, Toso A, Dabizzi RP. Increase of myocardial salvage and left ventricular function recovery with intracoronary abciximab downstream of the coronary occlusion in patients with acute myocardial infarction treated with primary coronary intervention. Catheter Cardiovasc Interv. 2004;62:186-192.

36. Iancu A, Ober C, Bondor CI, Cadiş H. Microvascular effect of intracoronary eptifibatide in acute myocardial infarction. Cardiology. 2012;123:46-53. 